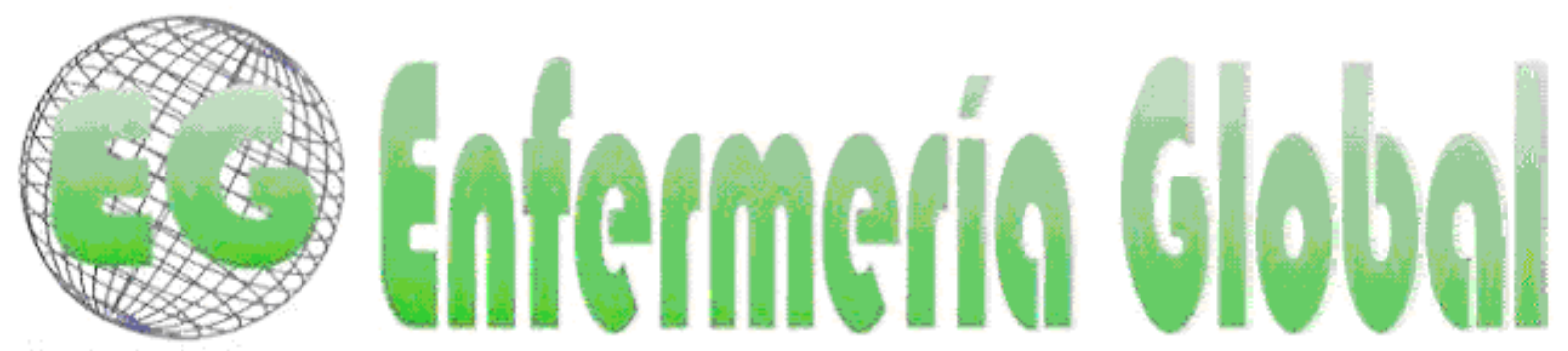

\title{
Capacidad de agencia de autocuidado y factores relacionados con la agencia en personas con insuficiencia cardíaca de la ciudad de Medellín (Colombia)
}

Self-care agency ability and factors related to the agency in people with heart failure of Medellin (Colombia)

\section{${ }^{*}$ Rodríguez Gázquez, $M^{a}$ de los Ángeles **Arredondo Holguín, Edith ***Salamanca Acevedo, Yurany Andrea}

\begin{abstract}
*Profesora Doctora de la Facultad de Enfermería. E-mail: mariangelesrodriguezg@hotmail.com **Magíster en Enfermería. Profesora de la Facultad de Enfermería ***Estudiante de Enfermería y miembro del semillero de investigación Kairos de la Facultad de Enfermería. Universidad de Antioquía. Colombia.
\end{abstract}

Palabras clave: insuficiencia cardíaca; autocuidado; educación en enfermería; teoría de enfermería; investigación en enfermería

Keywords: heart failure; self-care; education; nursing; nursing theory; nursing research.

\section{RESUMEN}

Objetivo. Explorar la asociación entre la capacidad de agencia de autocuidado con algunos factores básicos condicionantes relacionados con la agencia en personas con IC de la ciudad de Medellín (Colombia).

Material y Método. Estudio de corte transversal que se llevó a cabo en una muestra por conveniencia de 266 personas con IC de dos instituciones hospitalarias de la ciudad de Medellín (Colombia) desde 2007 a 2011. Se utilizó un instrumento en el que se incluyeron variables sociodemográficas, de apoyo social y clínicas. Para la evaluación de la capacidad de agencia de autocuidado se empleó la versión de la Appraisal of Self-care Agency Scale-ASA- de Evers, validada al castellano por Gallegos.

Resultados. La capacidad de agencia de autocuidado fue deficiente en el $47,0 \%$ de los participantes. Los mayores puntajes de ASA se encontraron en las personas de $\geq 50$ años, en los niveles socioeconómicos más altos, en los casados, en los que tenían educación tecnológica o universitaria, los que practicaban actividades recreativas, los que tenían cualquier tipo de apoyo social, estaban en clase funcional I y en la fracción de eyección de $\leq 40 \%$. En análisis de regresión logística mostró que la agencia de autocuidado suficiente está relacionada con la fracción de eyección, la edad y el estado civil. 
Conclusión. Fue deficiente la capacidad de agencia de autocuidado en la mitad de los participantes. Los factores básicos condicionantes, como la fracción de eyección, edad y estado civil, están asociados a una mejor capacidad de agencia de autocuidado.

\section{ABSTRACT}

Objective: To explore the association between the ability of self-care agency with some basic conditioning factors related to the agency in people with heart failure of Medellin (Colombia)

Material and methods: A cross-sectional study was conducted in a convenience sample of 266 people with heart failure of two hospitals in the city of Medellín (Colombia) from 2007 to 2011. An instrument which included socio-demographic variables, social support and clinics was used. To evaluate the ability of self-care agency was used the version of the Appraisal of Self-care Agency Scale-ASA of Evers, validated in Spanish by Gallegos.

Results: The self-care agency ability was deficient in $47,0 \%$ of the participants. The higher ASA scores were found in people $\geq 51$ years old, higher socioeconomic levels, married, technical or university education, those who practiced leisure activities, with any kind of social support and those with functional class I and ejection fraction of $\leq 40 \%$. Logistic regression analysis showed that sufficient selfcare agency is related to ejection fraction, age and marital status.

Conclusion: The self-care agency ability was deficient in the middle of the participants. Basic conditioning factors, like ejection fraction, age and marital status, were associated with better self-care agency ability.

\section{INTRODUCCIÓN}

La Insuficiencia Cardiaca (IC) es una enfermedad prevalente en todo el mundo ${ }^{(1)}$. Las personas con esta enfermedad sufren continuamente de ciclos de retención de líquidos, exacerbaciones de síntomas y hospitalizaciones, llevando además a la pérdida progresiva de la capacidad de cuidarse ${ }^{(2)}$. Dichas complicaciones en el estado de salud son posibles de prevenir si se tienen adecuadas conductas de autocuidado $^{(3)}$.

Se sabe que solo uno de cada dos pacientes con IC se pesa, controla la ingestión y eliminación de líquidos ${ }^{(4)}$ tiene dietas bajas en sodio ${ }^{(5)}$ y, aunque reconoce los síntomas asociados al deterioro de su enfermedad (dificultad para respirar, fatiga, entre otros), tiene dificultades para buscar atención temprana ${ }^{(6)}$. Por consiguiente, mejorar el autocuidado es un resultado importante en el cuidado enfermero que se brinde a la persona con $\mathrm{IC}^{(7)}$.

Etimológicamente el autocuidado está conformado por las palabras: auto del griego aúro que significa "por uno mismo", y cuidado del latín cogitātus que significa "pensamiento", lo que sería entonces el cuidado propio. Este constructo es muy utilizado en la disciplina enfermera, desde que Dorotea Orem ${ }^{(8)}$ lo conceptualizó en la Teoría General de Enfermería, la cual tiene tres subteorías (autocuidado, déficit de autocuidado y sistemas de enfermería).

El concepto de esta teorista es que el autocuidado es el conjunto de acciones intencionadas y aprendidas que realiza la persona para controlar los factores internos o externos, que pueden comprometer su vida y desarrollo posterior; mientras que el concepto capacidad de agencia de autocuidado lo define como la cualidad, aptitud o habilidad de la persona que le permite realizar una acción intencionada para 
participar en el autocuidado; habilidad que es desarrollada en el curso de la vida diaria por medio de un proceso espontáneo de aprendizaje que incluye: atender, entender, regular, adquirir conocimientos, tomar decisiones y actuar ${ }^{(9)}$.

La agencia de autocuidado es descrita como una estructura compleja de capacidades fundamentales que agrupa las habilidades básicas del individuo (sensación, percepción, memoria y orientación), los componentes de poder (capacidades específicas relacionadas con la habilidad del individuo para comprometerse en el autocuidado) $\mathrm{y}$, las capacidades para operaciones de autocuidado $^{(10)}$. Las operaciones de esta última capacidad, Gast et al. $^{(10)}$ las subdivide en tres tipos: 1) estimativas: se evalúan las condiciones y factores individuales y del medio ambiente que son importantes para su autocuidado; 2) transicionales: se toman decisiones sobre lo que se debe y puede hacer para mejorarlo; y 3) productivas: se realizan las medidas para satisfacer los requisitos de autocuidado.

Orem denominó factores básicos condicionantes (FBC) a los factores internos y externos que modifican la capacidad de la persona para cuidarse de sí, entre ellos: la edad, el sexo, la etapa evolutiva de desarrollo, los patrones de vida, las condiciones socioeconómicas, los factores socioculturales, el sistema familiar, el medio ambiente, el estado de salud y el sistema de salud ${ }^{(10)}$.

Para la disciplina enfermera ha sido de interés contar con instrumentos validados y con buen poder psicométrico que sean capaces de detectar cambios en la capacidad de agencia de autocuidado de los pacientes ${ }^{(11)}$. Dentro de las escalas que se han empleado a nivel mundial en individuos con diferentes enfermedades crónicas ${ }^{(12)}$ que requieran el desarrollo de capacidades específicas para el mejoramiento de las actividades de autocuidado, se encuentra el Appraisal of Self-care Agency Scale ASA- de Evers ${ }^{(13)}$, instrumento que mide el poder de los componentes del autocuidado en individuos de población general y que está basada en la Teoría del Déficit de Autocuidado de Orem ${ }^{(9)}$. La ASA fue traducida y validada al español por Gallegos ${ }^{(14)}$ en pacientes con IC, mostrando una confiabilidad para población mexicana de 0,77.

En Colombia, se realizó la segunda versión de ASA en español por Velandia y Rivera ${ }^{(15)}$ en personas con factores de riesgo cardiovascular, teniendo esta segunda escala en castellano una confiabilidad de 0,74 y adecuada validez de constructo, mostrando el análisis factorial la presencia de nueve factores con posibilidad de interpretación desde el punto de vista clínico ${ }^{(16)}$.

El objetivo de este estudio fue explorar la asociación entre la capacidad de agencia de autocuidado con algunos factores básicos condicionantes relacionados con la agencia en personas con IC que asistían a dos instituciones hospitalarias de la ciudad de Medellín (Colombia).

\section{MATERIAL Y MÉTODO}

Estudio de corte transversal en el que se seleccionó por conveniencia una muestra de 266 personas que estaban siendo tratadas por IC en dos instituciones hospitalarias de la ciudad de Medellín desde 2007 a 2011. Los participantes debían cumplir con los siguientes criterios de inclusión: ser mayor de edad, tener diagnóstico médico de falla cardiaca con clase funcional I, II o III compensada, sin alteración del estado mental y, firmar el consentimiento informado. La información suministrada por los pacientes fue tratada con confidencialidad y se salvaguardó el secreto profesional. 
En este estudio se utilizó un cuestionario que contenía la siguiente información: a) características sociodemográficas: edad, sexo, estado civil, ocupación y nivel de estudios; b) redes de apoyo social: familia, amigos e institución de salud; c) variables clínicas: comorbilidad, clase funcional y fracción de eyección; y, d) evaluación de la capacidad de agencia de autocuidado: se empleó la versión de la ASA validada por Gallegos $^{(14)}$, que se compone de 24 ítems con cinco opciones de respuesta tipo Likert ( 1 = nunca, $2=$ rara vez, $3=$ a veces, $4=$ a menudo y $5=$ siempre $)$. El puntaje máximo que se puede alcanzar es de 120 , teniendo la precaución de reversar para la puntación total los ítems 6, 11 y 20 que son negativos.

La base de datos y el análisis estadístico de la información se realizaron en el programa SPSS versión 18.0. Se hicieron los siguientes análisis: a) univariado para la descripción de las características generales de la muestra; b) bivariado con el fin de relacionar la agencia de autocuidado con las variables sociodemográficas y clínicas de los pacientes con la ayuda de las pruebas, $t$ de Student y $F$ estadística; y c) regresión logística binaria en la que se analizó la capacidad de agencia de autocuidado con relación a las principales variables explicativas incluidas en el estudio. Se dicotomizó el puntaje total de la escala, estableciendo por acuerdo de investigadores el punto de corte de 90 puntos, que correspondería al cuartil más alto, definiendo la capacidad de autocuidado como 'suficiente' $(\geq 89$ puntos $=1)$ o 'insuficiente' ( $\leq 88$ puntos $=0$ ). Para los parámetros en el modelo se consideró como criterio de inclusión el tener una probabilidad entre 0,05 y 0,19. El modelo primero incluyó una selección inicial de covariables relacionadas con la variable dependiente teniendo en cuenta la literatura y las relaciones estadísticas encontradas en el análisis bivariado. Se utilizó el método Enter para la selección final de variables; y, d) consistencia interna por medio del alfa de Cronbach. En todos los análisis estadísticos se consideró asociación estadísticamente significativa los valores de probabilidad inferiores a 0,05 .

En este estudio se evaluó la consistencia interna del ASA, encontrándose un alfa de Cronbach ajustado de: 0,69 el cual puede considerarse aceptable ${ }^{(17)}$.

\section{RESULTADOS}

En este estudio participaron 266 pacientes con IC. En la Tabla 1 se aprecian los factores básicos condicionantes de tipo sociodemográfico que, en términos generales, fueron las siguientes: la media de edad fue de 62,1 años (mínima 19 y máxima de 94), el $62,0 \%$ son de sexo femenino, los niveles socioeconómicos 2 y 3 fueron los de mayor proporción (67,3\%), predomina el estado civil casado (67,3\%), uno de cada dos tenía formación académica de primaria y menos y uno de cada tres está empleado. En cuanto a las redes sociales de los participantes, la familiar es la más frecuente $(91,4 \%)$, seguida por la institucional $(13,2 \%)$.

Pasando a las variables clínicas, también en la Tabla 1 se aprecia que la media en la fracción de eyección de los pacientes con esta enfermedad fue de 31,1\%, la mitad de los pacientes tuvieron clase funcional I, y la hipertensión arterial y la Diabetes Mellitus fueron las principales comorbilidades (56,0\% y $41,7 \%$, respectivamente). 
Tabla 1. Factores básicos condicionantes en 266 pacientes con IC

\begin{tabular}{|c|c|}
\hline Característica & Valor \\
\hline Edad en años (media $\pm D E$ ) & $62,1 \pm 13,4$ \\
\hline \multicolumn{2}{|l|}{ Sexo } \\
\hline Femenino $(n, \%)$ & $165(62,0)$ \\
\hline Masculino $(n, \%)$ & $101(38,0)$ \\
\hline \multicolumn{2}{|l|}{ Nivel socioeconómico $(\mathrm{n}, \%)$} \\
\hline 1 & $13(4,9)$ \\
\hline 2 & $68(25,6)$ \\
\hline 3 & $111(41,7)$ \\
\hline 4 & $37(13,9)$ \\
\hline 5 & $31(11,7)$ \\
\hline 6 (El más alto) & $6(2,3)$ \\
\hline \multicolumn{2}{|l|}{ Estado civil $(\mathrm{n}, \%)$} \\
\hline Soltero & $28(10,5)$ \\
\hline Casado & $179(67,3)$ \\
\hline Separado & $15(5,6)$ \\
\hline Viudo & $42(15,8)$ \\
\hline \multicolumn{2}{|l|}{ Formación académica $(n, \%)$} \\
\hline Ninguna & $20(7,5)$ \\
\hline Primaria & $122(45,9)$ \\
\hline Secundaria & $41(15,4)$ \\
\hline Técnica & $32(12,0)$ \\
\hline Tecnóloga & $24(9,0)$ \\
\hline Universitaria & $27(10,2)$ \\
\hline \multicolumn{2}{|l|}{ Redes de apoyo $(n, \%)$} \\
\hline Ninguna & $6(2,3)$ \\
\hline Familiar & $243(91,4)$ \\
\hline Amigos & $9(3,4)$ \\
\hline Institucional & $35(13,2)$ \\
\hline \multicolumn{2}{|l|}{ Actividad actual principal $(\mathrm{n}, \%)$} \\
\hline Sin actividad & $46(17,3)$ \\
\hline Oficios domésticos & $74(27,8)$ \\
\hline Empleado & $91(34,2)$ \\
\hline Actividades recreativas & $55(20,7)$ \\
\hline \multicolumn{2}{|l|}{ Clase funcional NYHA $(\mathrm{n}, \%)$} \\
\hline I & $133(50,0)$ \\
\hline II & $94(35,3)$ \\
\hline III & $39(14,7)$ \\
\hline 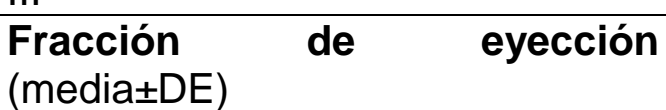 & $31,1 \% \pm 10,3 \%$ \\
\hline \multicolumn{2}{|l|}{ Comorbilidad $(\mathrm{n}, \%)$} \\
\hline Hipertensión arterial & $149(56,0)$ \\
\hline Diabetes Mellitus & $111(41,7)$ \\
\hline Enfermedad cardíaca congestiva & $58(21,9)$ \\
\hline
\end{tabular}

En el estudio, la media del puntaje total de la escala fue $89,3 \pm 10.0$, con un valor mínimo de 57 y un máximo de 110. Según el punto de corte de 90, definido 
previamente en la metodología, la capacidad de agencia de autocuidado fue deficiente en el $47,0 \%$ de los casos y suficiente en el $53,0 \%$ restante.

En la Tabla 2 se aprecia que, en una escala de uno a cinco, los ítems mejor puntuados fueron, con 4,5: 'Si no puedo cuidarme puedo buscar ayuda' y 'Puedo sacar tiempo para mí'; con 4,4: 'Hago en primer lugar lo que sea necesario para mantenerme con salud', 'Soy capaz de tomar medidas para garantizar que mi familia y yo no corramos peligro', 'Soy capaz de evaluar qué tanto me sirve lo que hago para mantenerme con salud' y 'A pesar de las limitaciones para movilizarme soy capaz de cuidarme como a mí me gusta'; y, con 4,3: 'Puedo hacer lo necesario para mantener limpio el ambiente donde vivo' y 'He sido capaz de cambiar hábitos que tenía muy arraigados con tal de mejorar mi salud'.

Por el contrario, los ítems con peores puntajes fueron, con 3,3: 'Cuando necesito ayuda puedo recurrir a mis amigos de siempre' y 'Cuando tengo que tomar una nueva medicina cuento con una persona que me brinda información sobre los efectos secundarios'; con 3,2: 'Cambio la frecuencia con que me baño para mantenerme limpio'; y con 3,1: 'Puedo buscar mejores formas para cuidar mi salud que las que tengo ahora' y 'Pienso en hacer ejercicio y descansar un poco durante el día, pero no llego a hacerlo'.

Tabla 2. Valor de la media y desviación estándar (DE) por ítem de la escala ASA en 266 pacientes con IC

Ítem

Promedio $\pm D E$

ASA01. A medida que cambian las circunstancias voy haciendo ajustes para mantenerme con salud

ASA 02. Reviso si son buenas las formas que practico habitualmente para mantenerme con salud

ASA03. Si tengo problemas para moverme o desplazarme me las arreglo para conseguir ayuda

ASA04. Puedo hacer lo necesario para mantener limpio el ambiente donde vivo

ASA05. Hago en primer lugar lo que sea necesario para mantenerme con salud

ASA06. Me faltan las fuerzas necesarias para cuidarme como debo

ASA07. Puedo buscar mejores formas para cuidar mi salud que las que tengo ahora

ASA08. Cambio la frecuencia con que me baño para mantenerme limpio

ASA09. Para mantener el peso que me corresponde hago cambios en mis hábitos alimenticios

ASA10. Cuando hay situaciones que me afectan, las manejo de manera que pueda mantener mi forma de ser

ASA11. Pienso en hacer ejercicio y descansar un poco durante el día, pero no llego a hacerlo

ASA12. Cuando necesito ayuda puedo recurrir a mis amigos de siempre

$4,2 \pm 1,1$

$4,2 \pm 1,2$

$3,6 \pm 1,7$

$4,3 \pm 1,2$

$4,4 \pm 0,9$

$3,5 \pm 1,5$

$3,1 \pm 1,7$

$3,2 \pm 1,8$

$3,8 \pm 1,4$

$3,8 \pm 1,3$

$3,1 \pm 1,5$

$3,3 \pm 1,7$ 


\begin{tabular}{|l|c|}
\hline ASA13. Puedo dormir lo suficiente como para sentirme descansado & $3,9 \pm 1,2$ \\
\hline $\begin{array}{l}\text { ASA14. Cuando tengo información sobre mi salud pido explicaciones } \\
\text { sobre lo que no entiendo }\end{array}$ & $4,1 \pm 1,4$ \\
\hline ASA15. Examino mi cuerpo para ver si hay algún cambio & $3,5 \pm 1,5$ \\
\hline $\begin{array}{l}\text { ASA16. He sido capaz de cambiar hábitos que tenía muy arraigados } \\
\text { con tal de mejorar mi salud }\end{array}$ & $4,3 \pm 1,0$ \\
\hline $\begin{array}{l}\text { ASA17. Cuando tengo que tomar una nueva medicina cuento con } \\
\text { una persona que me brinda información sobre los efectos } \\
\text { secundarios }\end{array}$ & $3,3 \pm 1,7$ \\
\hline $\begin{array}{l}\text { ASA18. Soy capaz de tomar medidas para garantizar que mi familia } \\
\text { y yo no corramos peligro }\end{array}$ & $4,4 \pm 1,0$ \\
\hline $\begin{array}{l}\text { ASA19. Soy capaz de evaluar qué tanto me sirve lo que hago para } \\
\text { mantenerme con salud }\end{array}$ & $4,4 \pm 1,0$ \\
\hline $\begin{array}{l}\text { ASA 20. Debido a mis ocupaciones diarias me resulta difícil sacar } \\
\text { tiempo para cuidarme }\end{array}$ & $3,6 \pm 1,6$ \\
\hline $\begin{array}{l}\text { ASA21. Si mí salud se ve afectada yo puedo conseguir la } \\
\text { información necesaria sobre qué hacer }\end{array}$ & $4,1 \pm 1,3$ \\
\hline ASA22. Si no puedo cuidarme puedo buscar ayuda & $4,5 \pm 1,0$ \\
\hline $\begin{array}{l}\text { ASA23. Puedo sacar tiempo para mí } \\
\text { ASA24. A pesar de las limitaciones para movilizarme, soy capaz de } \\
\text { cuidarme como a mí me gusta }\end{array}$ & $4,5 \pm 1,0$ \\
\hline
\end{tabular}

En la Tabla 3 se puede apreciar la diferencia del puntaje de ASA para las categorías de algunos factores básicos condicionantes de interés. Las mayores medias en el puntaje total de la escala de agencia se encontraron en las personas de 51 y más años, en los niveles socioeconómicos 4 y 5 , en los casados, en los que tenían educación tecnológica o universitaria, los que practicaban actividades recreativas y en los que tienen cualquier tipo de apoyo social.

En cuanto a las variables clínicas, el puntaje fue mayor en la clase funcional I y en la fracción de eyección de $40 \%$ y menos. Las únicas diferencias de medias de puntaje de ASA que fueron estadísticamente significativas entre las categorías de las variables de interés fueron las de actividad actual y clase funcional.

Tabla 3. Valores de puntuación promedio, mínimo y máximo de las categorías de los factores básicos condicionantes

\begin{tabular}{|c|c|c|c|c|c|}
\hline Característica & Media $\pm \mathrm{DE}$ & Mínimo & Máxima & $\begin{array}{l}\text { Prueba } \\
\text { F }\end{array}$ & $\begin{array}{l}\text { Valor de } \\
p\end{array}$ \\
\hline \multicolumn{6}{|c|}{ Edad en años (media $\pm D E)$} \\
\hline$\leq 40$ & $86,3 \pm 7,2$ & 70 & 96 & 0,75 & 0,56 \\
\hline $41-50$ & $87,8 \pm 10,5$ & 67 & 104 & & \\
\hline $51-60$ & $90,4 \pm 10,1$ & 62 & 107 & & \\
\hline $60-69$ & $89,1 \pm 10,5$ & 57 & 110 & & \\
\hline$\geq 70$ & $89,9 \pm 10,0$ & 71 & 109 & & \\
\hline \multicolumn{6}{|l|}{ Sexo } \\
\hline Masculino & $89,2 \pm 10,8$ & 57 & 110 & 0,20 & 0,11 \\
\hline Femenino & $89,6 \pm 8,7$ & 70 & 104 & & \\
\hline
\end{tabular}




\begin{tabular}{|c|c|c|c|c|c|}
\hline \multicolumn{6}{|l|}{ Nivel socioeconómico } \\
\hline 1 & $88,0 \pm 11,9$ & 70 & 110 & 1,72 & 0,12 \\
\hline 2 & $87,9 \pm 10,8$ & 57 & 104 & & \\
\hline 3 & $88,4 \pm 9,6$ & 67 & 109 & & \\
\hline 4 & $91,1 \pm 10,4$ & 71 & 107 & & \\
\hline 5 & $93,2 \pm 8,7$ & 73 & 107 & & \\
\hline 6 (el más alto) & $88, \pm 4,3$ & 83 & 93 & & \\
\hline \multicolumn{6}{|l|}{ Estado civil } \\
\hline Soltero & $90,7 \pm 7,8$ & 71 & 104 & 0,37 & 0,83 \\
\hline Casado & $89,4 \pm 10,6$ & 57 & 110 & & \\
\hline Separado & $90,2 \pm 10,1$ & 74 & 104 & & \\
\hline Viudo & $87,9 \pm 9,2$ & 70 & 103 & & \\
\hline \multicolumn{6}{|l|}{ Formación académica } \\
\hline Ninguna & $86,6 \pm 10,5$ & 71 & 107 & 1,57 & 0,16 \\
\hline Primaria & $88,8 \pm 9,8$ & 57 & 110 & & \\
\hline Secundaria & $89,7 \pm 9,9$ & 67 & 104 & & \\
\hline Técnica & $88,1 \pm 11,6$ & 71 & 109 & & \\
\hline Tecnóloga & $89,9 \pm 9,6$ & 67 & 107 & & \\
\hline Universitaria & $93,8 \pm 8,1$ & 74 & 105 & & \\
\hline \multicolumn{6}{|c|}{ Actividad actual principal } \\
\hline Sin actividad & $85,9 \pm 9,8$ & 68 & 110 & 3,56 & 0,01 \\
\hline Oficios domésticos & $89,1 \pm 9,3$ & 70 & 104 & & \\
\hline Empleado & $89,4 \pm 10,7$ & 57 & 109 & & \\
\hline Actividades recreativas & $92,4 \pm 9,2$ & 67 & 107 & & \\
\hline \multicolumn{6}{|l|}{ Redes de apoyo } \\
\hline \multicolumn{6}{|l|}{ Apoyo familiar } \\
\hline $\mathrm{Si}$ & $91,0 \pm 8,6$ & 57 & 104 & 0,70 & 0,40 \\
\hline No & $89,1 \pm 10,2$ & 72 & 110 & & \\
\hline \multicolumn{6}{|l|}{ Apoyo de amigos } \\
\hline $\mathrm{Si}$ & $91,3 \pm 10,7$ & 72 & 103 & 0,37 & 0,54 \\
\hline No & $89,2 \pm 10,0$ & 57 & 110 & & \\
\hline \multicolumn{6}{|l|}{ Apoyo institucional } \\
\hline $\mathrm{Si}$ & $90,6 \pm 9,6$ & 68 & 104 & 0,65 & 0,42 \\
\hline No & $89,1 \pm 10,1$ & 57 & 110 & & \\
\hline \multicolumn{6}{|l|}{ Clase funcional NYHA } \\
\hline l & $90,9 \pm 10,2$ & 57 & 109 & 3,65 & 0,02 \\
\hline II & $87,9 \pm 10,0$ & 62 & 110 & & \\
\hline III & $87,1 \pm 8,6$ & 71 & 104 & & \\
\hline \multicolumn{6}{|l|}{ Fracción de eyección } \\
\hline$\leq 40$ & $92,3 \pm 8,8$ & 70 & 105 & 2,27 & 0,08 \\
\hline $41-50$ & $89,5 \pm 10,2$ & 57 & 110 & & \\
\hline $51-60$ & $83,9 \pm 9,9$ & 68 & 100 & & \\
\hline 61 y más & $85,7 \pm 9,3$ & 77 & 98 & & \\
\hline
\end{tabular}


En el análisis de regresión logística, se seleccionó un modelo que presentó una aceptable clasificación general $(62,7 \%)$, con una prueba de bondad del ajuste de Hosmer y Lemeshow que aceptaba la hipótesis nula que decía que la distribución teórica del modelo era igual a la observada $\left(X^{2}=2,38, p=0,88\right)$, y la prueba ómnibus del modelo final rechazó la hipótesis nula de que todos los coeficientes $B_{i}$ eran iguales a cero $\left(X^{2}=15,34, p=0,02\right)$. Se aprecia en la Tabla 4 que la agencia de autocuidado suficiente está relacionada finalmente con los factores básicos condicionantes de: fracción de eyección, la edad y el estado civil.

Tabla 4. Resultados de la regresión logística binaria para adecuada agencia de autocuidado en pacientes con falla cardíaca

\begin{tabular}{|l|c|c|c|c|c|c|c|}
\hline Parámetro & B & $\begin{array}{c}\text { Error } \\
\text { típico }\end{array}$ & Wald & $\begin{array}{c}\text { Valor de } \\
\mathbf{p}\end{array}$ & OR & \multicolumn{2}{|c|}{ IC $_{95 \%}$ OR } \\
\hline $\begin{array}{l}\text { Fracción de } \\
\text { eyección }\end{array}$ & $-0,03$ & 0,01 & 4,32 & 0,03 & 0,97 & 0,95 & 0,99 \\
\hline Edad & 0,04 & 0,01 & 9,72 & 0,01 & 1,04 & 1,01 & 1,06 \\
\hline Estado civil & $-0,43$ & 0,18 & 5,51 & 0,01 & 0,65 & 0,46 & 0,93 \\
\hline Constante & $-0,31$ & 0,73 & 0,17 & 0,67 & 0,73 & & \\
\hline
\end{tabular}

\section{DISCUSIÓN}

En este estudio de 266 pacientes con IC el 53\% de ellos tuvieron capacidad de agencia de autocuidado suficiente, cifra mucho más alta a la reportada por otros estudios en Colombia ${ }^{(18)}$ en pacientes hipertensos (47\%) y en personas con factores de riesgo cardiovasculares $(36 \%)^{(15)}$.

Con respecto a los ítems del ASA mejor puntuados, encontramos diferencias con el estudio de Rivera con pacientes hipertensos ${ }^{(18)}$ en algunos ítems que, en nuestros pacientes tuvieron las más altas medias, mientras que en el estudio referenciado fueron de los peores: Puedo sacar tiempo para mí y ‘Puedo hacer lo necesario para mantener limpio el ambiente donde vivo'. Situación contraria se observó con los ítems: 'Cuando necesito ayuda puedo recurrir a mis amigos de siempre' y 'Para mantener el peso que me corresponde hago cambios en mis hábitos alimenticios'. En el resto de ítems el comportamiento fue muy similar. La razón de dichas divergencias puede ser explicada por el hecho de que los pacientes del estudio de Rivera estaban internados, lo que habla de un deterioro posible en ciertas conductas de autocuidado los podría haber llevado a la hospitalización.

En cuanto a las diferencias en la puntuación de la ASA según los factores básicos condicionantes que se abordaron en este estudio, se apreció que a mayor edad era mejor el puntaje de agencia de autocuidado, relación que fue significativa en el modelo de regresión logística. Este hallazgo es similar al reportado por Artinian et al. ${ }^{(19)}$ cuando se asoció el aumento de la edad con una mayor probabilidad de realizar algunas conductas de autocuidado, entre ellas, acudir al médico cuando existen problemas de salud. Orem pensaba que la capacidad de agencia de autocuidado era diferente según la etapa evolutiva del individuo, por lo que estaba determinada directamente por la edad ${ }^{(20)}$. Similares resultados a los nuestros han sido relatados por Akyol et al. ${ }^{(21)}$, en pacientes hipertensos, y por Artinian et al. ${ }^{(19)}$, en personas con IC. 
No se apreció diferencia significante en nuestro estudio en la capacidad de agencia de autocuidado según sexo, lo que también fue encontrado en otras investigaciones $^{(19,22)}$, y que es diferente a los hallazgos de Velandia ${ }^{(15)}$ y de Abootalebi et al. ${ }^{(23)}$ en los que las mujeres tuvieron mejor capacidad de agencia de autocuidado, mientras que en el estudio de Friedman ${ }^{(24)}$ fueron los hombres.

Se sabe que el apoyo emocional de la pareja es un factor importante cuando hay que emprender cambios en los estilos de vida no saludables ${ }^{(25)}$. En este estudio, la capacidad de agencia de autocuidado fue mayor en los casados que en los viudos, siendo el estado civil una variable explicativa que se asoció significativamente a tener suficiente capacidad de agencia, siendo este hallazgo consistente con los resultados de otros cinco estudios ${ }^{(22,23,26-28)}$.

Las personas con IC que tenían estudios universitarios tuvieron los mayores puntajes de ASA. Lo anterior, es consistente con otra publicación sobre el tema, como la de Aboolatebi et al. ${ }^{(23)}$ en personas con IC iraníes. De acuerdo con Rockwell et al. ${ }^{(29)}$ los pacientes con más educación tienen mayor poder de juzgamiento a la hora de la toma de decisiones para mejorar las conductas de autocuidado. El nivel educativo, también se relaciona con el conocimiento que se tenga de la enfermedad, cuanto mayor sea éste, mejor podrá actuar el paciente de acuerdo con los cambios de los síntomas de la enfermedad que observa día a día ${ }^{(30)}$.

La ocupación es otro factor que puede afectar la capacidad de agencia de autocuidado. Los participantes que tenían empleo tuvieron mayor puntaje de ASA que las personas desempleadas, diferencia que fue también significante. Este resultado es consistente con el reportado en otros estudios en pacientes con IC ${ }^{(23,27,31)}$. También los datos mostraron que las personas que practicaban actividades recreativas como ocupación principal tuvieron el más alto nivel de capacidad de agencia; Riegel ${ }^{(32)}$ asocia estas actividades con la reducción del estrés, la que puede llevar a la reducción de la presión sanguínea y, que Wang ${ }^{(33)}$ además las ha relacionado a un mejor descanso nocturno, el cual es un factor de bienestar muy valorado en las personas mayores.

Coyle ${ }^{(34)}$ encontró que la ocupación no solo está directamente relacionada con la capacidad de autocuidado, sino que también se asocia a otras variables como el nivel educativo y el nivel socioeconómico, como efectivamente también se observó en nuestros datos y en el estudio iraní(23), en los cuales las personas con niveles socioeconómicos más altos tuvieron mejores puntajes de agencia de autocuidado.

Aunque sin ser significativamente diferentes, en nuestros datos el puntaje de la ASA fue mayor en aquellos pacientes tenían apoyo social (familiar, amigos e institucional). Se sabe que este apoyo amortigua los efectos adversos de la enfermedad. Las personas que cuentan con buenas redes sociales se adaptan más fácilmente a su enfermedad y mejoran la adherencia al tratamiento ${ }^{(35)}$. El apoyo social protege contra el estrés generado por la enfermedad y capacita al paciente para reevaluar la situación y adaptarse mejor a ella, ayudándole a desarrollar respuestas de afrontamiento ${ }^{(35)}$, por lo sería muy importante que se tuviera en cuenta a las personas que brindan este tipo de apoyo en el plan de cuidado de enfermería en los pacientes con IC.

Cuando se analizó la clase funcional ${ }^{(36)}$, fue estadísticamente significante dentro del modelo de la regresión logística el hallazgo de que a menor clase era mejor la 
capacidad de agencia de autocuidado. Esta situación también fue apreciada en los estudios de Abootalebi et al. ${ }^{(23)}$ y por Rockwell y Riegel ${ }^{(29)}$ y Friedman $^{(24)}$. Esto es explicable en el sentido de que a mayor clase funcional es peor el estado físico general del paciente y, por tanto, son menores las capacidades que tiene de cuidarse a sí mismo. Igual razonamiento podemos hacer con la fracción de eyección, los datos mostraron una relación inversa entre ésta y el puntaje de ASA, hallazgo que también fue observado por Lauck et al. ${ }^{(26)}$.

Tener adecuada agencia de autocuidado aumenta en la persona las probabilidades de adquirir o mejorar las conductas de salud, así como la capacidad específica de autogestión de su enfermedad ${ }^{(27)}$. Los pacientes pueden tener un buen puntaje de capacidad de agencia de autocuidado $y$, sin embargo, los niveles de agencia no siempre llevan a estas personas a la acción.

El cuidado de las personas con IC es un reto para el personal de salud ${ }^{(37)}$, pero especialmente para enfermería, pues su visión es fundamental para ayudar a los pacientes que sufren de esta enfermedad a llevar a cabo y mantener por sí mismos las conductas de autocuidado necesarias para conservar la salud y el bienestar.

\section{CONCLUSIÓN}

Los resultados de este estudio indicaron que la capacidad de agencia de autocuidado fue deficiente en casi la mitad de los participantes. El modelo de regresión logística mostró que los factores básicos condicionantes: fracción de eyección, edad y estado civil están asociados a una mejor capacidad de agencia de autocuidado.

\section{REFERENCIAS}

1. Gwadry-Sridhar FH, Flintoft V, Lee DS, Lee H, Guyatt GH. A systematic review and meta-analysis of studies comparing readmission rates and mortality rates in patients with heart failure. Arch Intern Med. 2004;164(21):2315-20.

2. Scott L, Setter-Kliner K, Britton A. The effects of nursing interventions to enhance mental health and quality of life among individuals with heart failure. Appl Nurs Res. 2004;17(4):248-56.

3. Bennett SJ, Sauve MJ, Shaw RMAcmocdichf. A conceptual model of cognitive deficits in chronic heart failure. J Nurs Scholarsh. 2005;37(3):222-8.

4.Moser DK, Doering LV, Chung ML. Vulnerabilities of patients recovering from an exacerbation of chronic heart failure. Am Heart J. 2005;150(5):984.e7-984.e13.

5. Hershberger R, Ni H, Nauman DJ, Burgess D, Toy W, Wise K, et al. Prospective evaluation of an outpatient heart failure management program designed for primary care: the Oregon model. J Card Fail. 2005;11(4):293-8.

6. Carlson B, Riegel B, Moser DK. Self-care abilities of patients with heart failure. Heart Lung. 2001;30(5):351-9.

7. López A, Guerrero S. Perspectiva internacional del uso de la teoría general de Orem. Invest Educ Enferm. 2006;24(2):90-100.

8. Orem D. Nursing: Concepts of Practice. St Louis, MO: Mosby; 1995.

9. Orem D. Normas prácticas en enfermería. Madrid: Ediciones Pirámide; 1983. p. 79115.

10. Gast HL, Denyes MJ, Campbell JC, Hartweg DL, Schott-Baer D, Isenberg M. Selfcare agency: conceptualizations and operationalizations. Adv Nurs Sci. 1989;12(1):2638. 
11. Jurgens CY, Fain JA, Riegel B. Psychometric testing of the heart failure somatic awareness scale. J Cardiovasc Nurs. 2006;21:95-102.

12. Sousa VD, Zauszniewski J, Bergquist-Beringer S, Musil C, Neese J, Jaber A. Reliability, validity and factor structure of the Appraisal of Self-Care Agency Scale Revised (ASAS-R). J Eval Clin Pract. 2010;16(6):1031-40.

13. Evers GCM. Appraisal of self-care agency: A.S.A. -Scale. The Netherlands: Van Gorcum \& Comp. B.V.; 1989.

14. Gallegos EC. Validez y confiabilidad de la versión en español de la escala: Valoración de las Capacidades de Autocuidado. Desarro Científ Enferm. 1998;6(9):260-6.

15. Velandia A, Rivera LN. Confiabilidad de la escala "Apreciación de la agencia de autocuidado" (ASA), segunda versión en español, adaptada para población colombiana. Av Enferm 2009;27(1):38-47.

16. Manrique-Abril F, Fernández A, Velandia A. Análisis factorial de la Escala Valoración de Agencia de Autocuidado (ASA) en Colombia. Aquichan. 2009;9(3):22235.

17. Oviedo HC, Campo A. Aproximación al uso del coeficiente alfa de Cronbach. Rev Colomb Psiquiatr. 2005;34(4):572-80.

18. Rivera LN. Capacidad de agencia de autocuidado en personas con hipertensión arterial hospitalizadas en una Clínica de Bogotá, Colombia. Rev Salud Pública. 2006;8(3):235-47.

19. Artinian NT, Magnan M, Sloan M, Lange MP. Self-care behaviors among patients with heart failure. Heart Lung. 2002;31(3):161-72.

20. Renpenning K, Taylor $S$. Self-care $t$ theory of nursing: Selected Papers of Dorothea Orem. New York: Springer Pub Co; 2003.

21. Akyol AD, Cetinkaya Y, Bakan G, Yarah S, Akkus SJocn. Self - care agency and factors related to this agency among patients with hypertension. J clin nurs. 2007; 16(4):679-87.

22. Heo S, Moser DK, Lennie TA, Riegel B, Chung ML. Gender differences in and factors related to self-care behaviors: a cross-sectional, correlational study of patients with heart failure. Int J Nurs Stud. 2008;45(12):1807-15.

23. Abootalebi G, Vosoghi N, Mohammadnejad E, Namadi M, Akbari M. Study of the self-care agency in patients with heart failure. Iran J Critical Care Nurs. 2012;4(4):2038.

24. Friedman MM. Gender differences in the health related quality of life of older adults with heart failure. Heart Lung. 2003;32(5):320-7.

25. Adams M, Bowden A, Humphrey D, McAdams L. Social support and health promotion lifestyles of rural women. Online J Rural Nurs Health Care. 2000;1 (1):28-40. 26. Lauck S, Johmson J, Ratner P. Self-Care behaviour and factors associated with patient outcomes following same-day discharge percutaneous coronary intervention. Eur J Cardiovasc Nurs 2009;8(3):190-9.

27. Sousa VD, Zauszniewski JA, Zeller RA, Neese JB. Factor analysis of the appraisal of self-care agency scale in American adults with diabetes mellitus. Diabetes Educ. 2008;34(1):98-108.

28. Sayers SL, Riegel B, Pawlowski S, Coyne JC, Samaha FF. Social support and self-care of patients with heart failure. Ann Behav Med. 2008;35(1):70-9.

29. Rockwell JM, Riegel B. Predictors of self-care in persons with heart failure. Heart Lung. 2001;30(1):18-25.

30. Riegel B, Vaughan Dickson V, Goldberg LR, Deatrick JA. Factors associated with the development of expertise in heart failure self-care. Nurs Res. 2007;56(4):235-43.

31. Oksel E, Akbiylk A, Kocak G. Self-care behavior analysis of patients with chronic heart failure. Eur J Cardiovasc Nurs. 2009;31(7):s22. 
32. Riegel B, Lee CS, Dickson VV. Self care in patients with chronic heart failure. Nat Rev Cardiol. 2011;8(11):644-54.

33. Wang T-J, Lee S-C, Tsay S-L, Tung H-H. Factors influencing heart failure patients' sleep quality. J Adv Nurs. 2010;66(8):1730-40.

34. Coyle RL. Information retrieval of self - care and dependent care agents using netwellness. Cincinnati: University of Cincinnati; 2000.

35. Davidson P. Issues in patient compliance. En: Millon TG, G. Meagher, R, editor. Handbook of clinical health of psychology. New York: Plenum Press; 1982. p. 417-34. 36. Association AH. Classes of Heart Failure AHA. [Internet] AHA; [acceso 19 de diciembre de 2011]. Disponible http://www.heart.org/HEARTORG/Conditions/HeartFailure/AboutHeartFailure/Classesof-Heart-Failure UCM 306328 Article.jsp\#.T00Zmlmk4dW.

37. Olivella M, Bonilla $P$, Bastidas CV. Fomento del autocuidado en la insuficiencia cardiaca. Enfermería Global. 2012;11(1):282-6. 